\title{
Topology and Origin of Effective Spin Meron Pairs in Ferromagnetic Multilayer Elements
}

\author{
Sebastian Wintz, ${ }^{1,2, *}$ Christopher Bunce, ${ }^{1, \dagger}$ Andreas Neudert, ${ }^{1}$ Michael Körner, ${ }^{1}$ Thomas Strache,,${ }^{1, \star}$ Matthias Buhl, ${ }^{1}$ \\ Artur Erbe, ${ }^{1}$ Sibylle Gemming, ${ }^{1,3}$ Jörg Raabe, ${ }^{4}$ Christoph Quitmann, ${ }^{4, \S}$ and Jürgen Fassbender ${ }^{1,2}$ \\ ${ }^{1}$ Helmholtz-Zentrum Dresden-Rossendorf, 01328 Dresden, Germany \\ ${ }^{2}$ Institut für Festkörperphysik, Technische Universität Dresden, 01069 Dresden, Germany \\ ${ }^{3}$ Technische Universität Chemnitz, 09107 Chemnitz, Germany \\ ${ }^{4}$ Swiss Light Source, Paul Scherrer Institut, 5232 Villigen, Switzerland
}

(Received 8 October 2012; published 22 April 2013)

\begin{abstract}
We report on pairs of converging-diverging spin vortices in $\mathrm{Co} / \mathrm{Rh} / \mathrm{NiFe}$ trilayer disks. The lateral magnetization distribution of these effective spin merons is directly imaged by means of element-selective $\mathrm{x}$-ray microscopy. By this method, both the divergence and circulation states of the individual layers are identified to be antisymmetric. Reversal measurements on corresponding continuous films reveal that biquadratic interlayer exchange coupling is the cause for the effective meron pair formation. Moreover, their three-dimensional magnetization structure is determined by micromagnetic simulations. Interestingly, the magnetic induction aligns along a flux-closing torus. This toroidal topology enforces a symmetry break, which links the core polarities to the divergence configuration.
\end{abstract}

DOI: 10.1103/PhysRevLett.110.177201

PACS numbers: $75.70 . \mathrm{Kw}, 75.70 . \mathrm{Cn}$, 75.75.Fk

Nontrivial topologies are highly relevant for many phenomena in physics, ranging from fundamental field theories [1] to condensed matter systems such as topological insulators [2] and high temperature superconductors [3]. In the context of magnetism, nontrivial spin textures exist, for example, as bubble [4] and vortex [5] states for which the topology is quantified by their Skyrmion number to \pm 1 and $\pm 1 / 2$, respectively [6]. As sketched in Fig. 1(a), a spin vortex consists of a planar magnetization $(\mathbf{M})$ curl, that tilts out of the plane in the nanoscopic core region $[7,8]$. There are two possible configurations for the curl's rotation sense (circulation $c$ ) and also two possible orientations for the core (polarity $p$ ):

$$
\begin{gathered}
p=\operatorname{sgn}\left[\mathbf{e}_{z} \cdot \mathbf{M}(r=0)\right], \\
c=\operatorname{sgn}\left\{\mathbf{e}_{z} \cdot[\mathbf{r} \times \mathbf{M}(r \neq 0)]\right\},
\end{gathered}
$$

resulting in two distinct vortex handednesses $h=c p$. Because of their chiral nature and their interesting dynamic properties, magnetic vortices have been intensely studied during the past decades [5,7-13]. In addition to fundamental aspects, these investigations have led to the proposal of vortex based memory cells [14] and spin-torque driven nano-oscillators [15-17]. For both concepts the integration of vortices into lateral arrays $[18,19]$ or multilayer systems is a crucial point [20-29].

A special spin state with nontrivial topology is the meron state [30-34]. It is realized by a radially in- or outwards pointing planar spin distribution, see Fig. 1(b), for whichcomplementary to the $c=0$ state-a divergence $(d)$ can be defined to

$$
d=\operatorname{sgn}\left[\mathbf{e}_{r} \cdot \mathbf{M}(r \neq 0)\right]
$$

Vortices and merons can be transformed into each other through a planar rotation of $\mathbf{M}(r)$ by $90^{\circ}$. In between these two extremes there is a state which is equivalent to the superposition of a meron with a purely tangential vortex. This state is referred to as an unconventional vortex [33] or effective meron. Experimentally, so far Phatak et al. have inferred the existence of such states from an approach combining Lorentz transmission electron microscopy with micromagnetic simulations [34]. Metastable pairs of effective merons with equal $c$ 's but opposing $d$ 's were identified in a trilayer disk which consisted of two ferromagnetic layers separated by a nonmagnetic spacer. The formation of these pairs was explained by assuming an antiferromagnetic (AFM) interlayer exchange coupling (IEC) [35] and a demagnetization energy barrier resulting from non-edgeparallel magnetization states.

In this Letter we now offer direct proof for the existence of effective meron pairs by means of layer-resolved magnetic imaging with scanning transmission $\mathrm{x}$-ray microscopy (STXM). Furthermore, we show that they can even be the lowest energy state of the system when a biquadratic [36-38] IEC contribution is present in the corresponding trilayer films. By applying micromagnetic simulations we derive that such effective merons have a three-dimensional spin structure, where the lateral divergence $\boldsymbol{\nabla} \mathbf{M}_{x y}$ is partially compensated by a vertical $\boldsymbol{\nabla} \mathbf{M}_{z}$. Interestingly, the resulting toroidal magnetization distribution leads to a topological symmetry break that enforces a strict relation between the $d$ and $p$ states of the system.

In order to create effective meron pairs, trilayer thin film disks with a radius of $R=1 \mu \mathrm{m}$ were fabricated; see sketch in Fig. 1(c). Patterning was achieved by a sequence of electron-beam lithography, thin film deposition via electron beam evaporation, and lift-off processing. The 


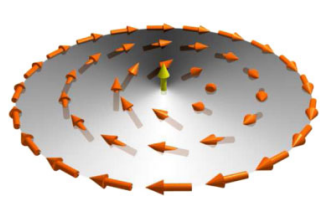

(a)

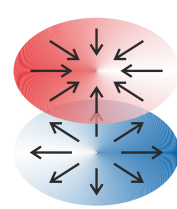

(b)

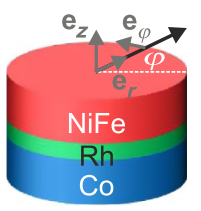

(c)
FIG. 1 (color online). Sketches of topological spin states in thin film elements. (a) Spin vortex with planar circulation and central core; magnetization orientation indicated by arrows. (b) Spin meron pair with diverging magnetization in the bottom layer and converging magnetization in the top layer. (c) Trilayer disk consisting of $\mathrm{Co}, \mathrm{Rh}$, and $\mathrm{NiFe}$.

$\mathrm{Co} / \mathrm{Rh} / \mathrm{NiFe}$ trilayer stack consists of two ferromagnetic layers $\left(\mathrm{Co}\right.$ and $\left.\mathrm{Ni}_{81} \mathrm{Fe}_{19}\right)$ of equal thickness $(t=50 \mathrm{~nm})$ and a nonmagnetic $\mathrm{Rh}$ interlayer in between [39]. For the thickness given $(t=0.8 \mathrm{~nm}), \mathrm{Rh}$ is expected to mediate a strong AFM IEC [40].

Figure 2 displays the remanent magnetization orientation $\left(\mathbf{m}_{x y}=\mathbf{M}_{x y} / M_{x y}\right)$ state of the Co layer (lower row) and the $\mathrm{NiFe}$ layer (upper row) of a $\mathrm{Co} / \mathrm{Rh} / \mathrm{NiFe}$ disk as measured by STXM [41]. Figures 2(a) and 2(b) show the STXM micrographs with sensitivity $m_{x}$ and $m_{y}$, respectively. The corresponding contrast scheme is indicated by the arrows in the gray scale wheels. An overlay of the micrographs 2(a) and 2(b) allows one to deduce the lateral magnetization distribution as sketched in 2(c). Obviously, each ferromagnetic layer exhibits an unconventional vortex. The average inclination of the planar $\mathbf{m}_{x y}$ with respect to the azimuthal unit vector $\left(\mathbf{e}_{\varphi}\right)$ is $\phi_{\mathrm{Co}}=-19^{\circ}$ and $\phi_{\mathrm{NiFe}}=117^{\circ}$, respectively, resulting in a planar coupling angle $\Phi=\arccos \left(\mathbf{m}_{x y}^{\mathrm{Co}} \mathbf{m}_{x y}^{\mathrm{NiFe}}\right)$ of $136^{\circ}$ (cf. Fig. 2, dashed lines) [42]. This distribution corresponds to a pair of effective spin merons with the following configuration: $c_{\mathrm{Co}}=+1, c_{\mathrm{NiFe}}=-1, d_{\mathrm{Co}}=+1$, and $d_{\mathrm{NiFe}}=-1$. Note that in contrast to $\mathrm{Co}, \phi_{\mathrm{NiFe}}$ is neither entirely constant

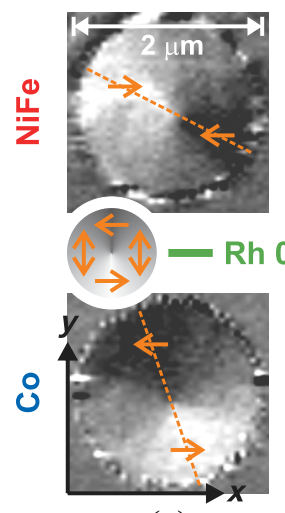

(a)

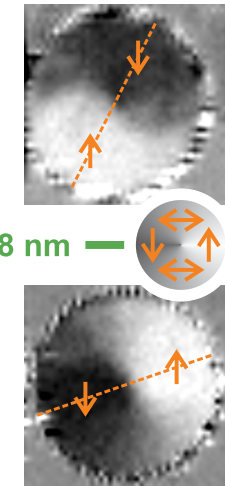

(b)
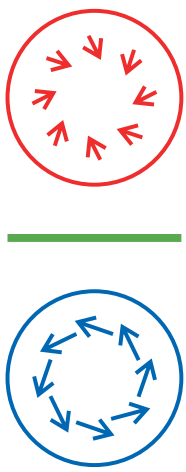

(c)

FIG. 2 (color online). Effective meron pairs in trilayer disks. (a), (b) STXM images showing the projected magnetization orientation $\mathbf{m}_{x}$ (a) and $\mathbf{m}_{y}$ (b) of the Co (bottom) and NiFe (top) layer with contrast as indicated (arrows). (c) Sketch of the resulting principle $\mathbf{M}_{x y}$ distribution.

over $r$ nor fully rotational symmetric. In particular, the $\mathbf{m}_{y}$ dominated regions are slightly more extended than those of $\mathbf{m}_{x}$. Nonetheless, this does not affect the principle state of the system, i.e., the average angle $\phi_{\mathrm{NiFe}}=117^{\circ}$, as well as the fact that $\mathbf{m}_{x y}$ runs through all planar orientations along $\varphi$ in a continuous and monotonic manner. We attribute the rotational asymmetry to sample imperfections as other effective meron structures were found to be symmetric in $\mathbf{m}_{x}$-sensitive measurements (not shown). In agreement with Ref. [34] the observed merons only occur in pairs, in particular, with opposing divergence $\left(d_{1}=-d_{2}\right)$. However, in contrast to Ref. [34], here also the circulations are antisymmetric $\left(c_{1}=-c_{2}\right)$. This is a crucial piece of evidence that reveals the existence of a noncollinear coupling in this system, namely, biquadratic IEC. Spin merons with symmetric circulations $\left(c_{1}=c_{2}\right)$ may exist as metastable states in purely bilinear AFM IEC systems [34]. However, they could not persist with antisymmetric circulations $\left(c_{1}=-c_{2}\right)$, as no energy barrier prevents the relaxation into the corresponding ground state of a purely tangential vortex pair [25].

IEC is described phenomenologically by the energy density per unit area,

$$
\sigma_{\mathrm{IEC}}=-J_{L} \frac{\mathbf{M}_{1} \cdot \mathbf{M}_{2}}{M_{1} M_{2}}-J_{Q} \frac{\left(\mathbf{M}_{1} \cdot \mathbf{M}_{2}\right)^{2}}{\left(M_{1} M_{2}\right)^{2}}
$$

which for the relative magnetic orientation of two ferromagnetic layers splits into a bilinear and a biquadratic contribution with the corresponding coupling constants $\left(J_{L}\right)$ and $\left(J_{Q}\right)$, respectively [36-38]. A negative (positive) $J_{L}$ favors an antiferromagnetic (ferromagnetic) alignment of the two layers, whereas a negative $J_{Q}$ energetically prefers their orthogonal $\left(\Phi=90^{\circ}\right)$ orientation.

In order to validate the explanation that a biquadratic IEC is responsible for the effective meron pair stability observed, we determined the IEC constants of a $\mathrm{Co}(25) / \mathrm{Rh}(0.8) /$ $\mathrm{NiFe}(25)(\mathrm{nm})$ continuous film [43]. The measured values of $J_{L}^{f}=-1.95 \mathrm{~mJ} / \mathrm{m}^{2}$ and $J_{Q}^{f}=-1.05 \mathrm{~mJ} / \mathrm{m}^{2}$ indeed reflect a strong biquadratic IEC contribution. However, the remanent coupling angle $\Phi_{0}^{f}$ resulting from these values is bigger than that observed in the effective meron pairs. This deviation is presumably a consequence of the altered interface roughnesses due to the different ferromagnetic layer thicknesses involved. The idea that a strong biquadratic IEC exists in this system is also supported by the response of the effective meron pair to quasistatic magnetic fields. For an external field of $\mu_{0} H=-15 \mathrm{mT}$ applied along the $x$ axis, we observed a layer-congruent displacement of the cores by about $200 \mathrm{~nm}$ [44]. This congruency can only be induced by IEC, since for purely dipolar coupled pairs the displacement would occur in different directions and by different magnitudes [27]. For the reasons given, we conclude that a biquadratic IEC is present in the trilayer structures and that it is necessary for the stability of the $c$-antisymmetric effective spin meron pairs observed. 
A perfectly planar spin meron pair $\left(\mathbf{M}_{z}=0\right.$ everywhere $)$ would be associated with a rather high demagnetization energy due to magnetic surface charges $\sim \mathbf{M} \cdot \mathbf{e}_{r}=$ $\pm M \pi R t$ at the cylinder jacket as well as magnetic volume charges $\sim \nabla \mathbf{M}= \pm M / r$ within the whole element, in particular, towards the center. Perpendicular magnetization components $M_{z}$ and their vertical variation $\left(\nabla \mathbf{M}_{z}\right)$ can lower this energy. The STXM experiment, however, is neither sensitive enough to detect small $M_{z}$ values nor capable of resolving axial changes of $\mathbf{M}$. Therefore, we carried out micromagnetic simulations in order to determine 3D magnetization structure of effective meron pairs [45]. A trilayer disk with size and parameters in accordance with the experiment was modeled on a $256 \times$ $256 \times(2+1+2)$ grid [46]. The initial magnetization states were defined to match those observed in the experiment with respect to $\Phi$. Stationary states were accessed by integrating the Landau-Lifshitz-Gilbert equation [47] assuming a strong damping $(\alpha=1)$.

Results from these micromagnetic simulations of effective meron pairs are shown in Fig. 3. The $x$ and $z$ projections of the magnetic orientation $\mathbf{m}$ are displayed in 3(a). Both ferromagnetic layers ( $\mathrm{Co}$ and $\mathrm{NiFe}$ ) were discretized into an inner and outer sublayer with respect to the $\mathrm{Rh}$ interlayer. The $m_{x}$ distributions [3(a), left] are in good agreement with the experimental results obtained by STXM [cf. Fig. 2(a)]. The deviations from $\mathbf{e}_{\phi}$ at $r=1 / 2 R$ are $\phi_{\mathrm{Co}}^{\text {sim }}=-33^{\circ}$ and $\phi_{\mathrm{NiFe}}^{\text {sim }}=106^{\circ}$ leading to $\Phi^{\text {sim }}=139^{\circ}$, which compares well with the experimental value of $\Phi=136^{\circ}$. The difference between the theoretical and experimental values of $\phi$ is most likely due to a variation of the ratio $M_{\mathrm{Co}} t_{\mathrm{Co}} / M_{\mathrm{NiFe}} t_{\mathrm{NiFe}}$ from the nominal value. Both ferromagnets (in particular $\mathrm{NiFe}$ ) exhibit a small variation of $\phi$ over $r$.

In order to visualize the $m_{z}$ distributions of the effective meron pair, the contrast scheme was enhanced by a factor of 5 in Fig. 3(a) (right). All sublayers show a dark contrast in the center and a bright contrast at the disk edge; i.e., $m_{z}$ is negative in the middle and positive at the outer parts. This feature is considerably more pronounced for both inner discretization layers of the disk relative to the corresponding outer ones. For equivalent discretization layers, the $m_{z}$ component of NiFe is slightly bigger than that of Co.

From the simulations we deduce that the 3D magnetization structure of an effective meron pair corresponds to a toroid that is superimposed with tangential vortex components. Figure 3(c) shows a cross-sectional sketch of such a toroidal spin distribution. While-apart from the core-the magnetization at the top and bottom face is almost oriented parallel to the film plane, it steadily tilts out of the plane towards the central interlayer. Along any vertical axis, the sign of $m_{z}$ is the same for Co and NiFe. Laterally, however, $m_{z}$ is changing sign from the edge to the center within each ferromagnet.

Quantitative values for the radial dependence of $m_{z}$ are given in Fig. 3(d) reflecting the toroidal structure. All the
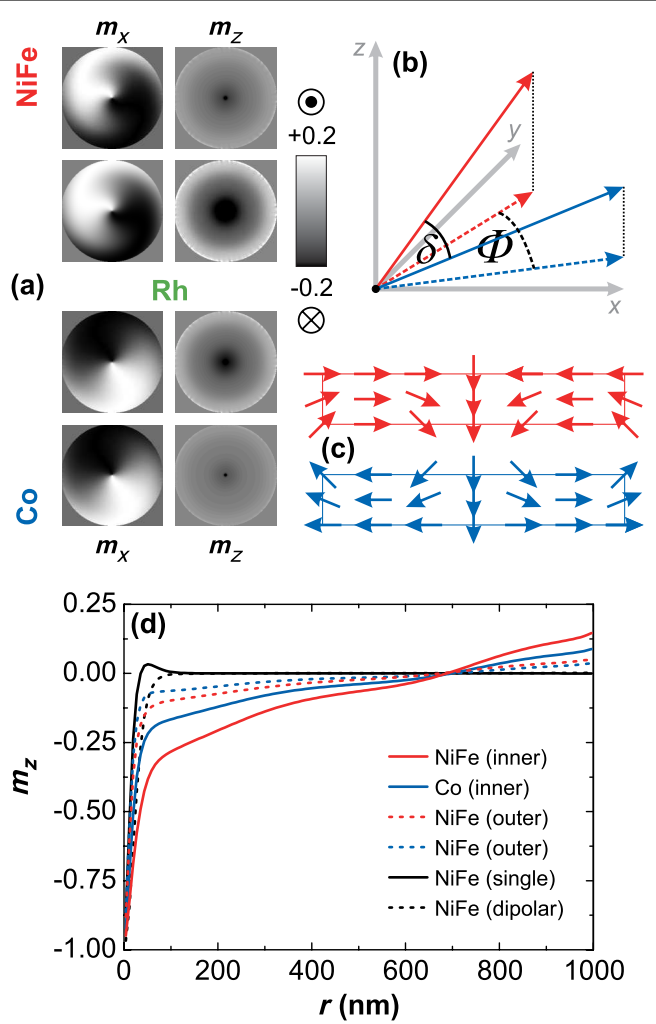

FIG. 3 (color online). Micromagnetic simulation of an effective meron pair. (a) $m_{x}$ and $m_{z}$ for a trilayer disk with dimensions as in the experiment, but reduced $J_{L}$. Both ferromagnets were discretized into an inner and outer sublayer. The $z$ contrast is enhanced $(\times 5)$ to visualize the toroidal magnetization structure. (b) Sketch of the three-dimensional coupling angle $\delta$ versus the planar coupling angle $\Phi$. (c) Cross-sectional sketch of the magnetization torus, neglecting the tangential components. (d) Radial dependence of $m_{z}$ for the inner and outer discretization layers of the effective meron, compared to a single NiFe layer and a $\mathrm{NiFe}$ layer of a dipolarly coupled vortex pair.

separate layers simulated within the meron pair exhibit a similar variation of $m_{z}$ with $r$. Starting from $m_{z}=-1$ at the center, the perpendicular component sharply decreases to values below -0.5 in the range of $r \sim 50 \mathrm{~nm}$. This feature can be seen as the actual core of the structure. At $r \approx 700 \mathrm{~nm}$ the $m_{z}$ distribution crosses zero and then monotonically increases to positive values below +0.25 at the sample edge. In contrast to the effective meron pairs, neither a single layer NiFe vortex nor the $\mathrm{NiFe}$ vortex of a dipolarly coupled vortex pair [48] exhibits any significant $m_{z}$ components for $r>100 \mathrm{~nm}$.

The $\phi_{i}^{\text {sim }}$ values also show a radial variation. At half the distance between center and edge, the planar coupling angle is $\Phi^{\text {sim }}=139^{\circ}$. It increases, however, to almost $180^{\circ}$ at the edge and close to the center, as $\phi_{\mathrm{Co}}^{\text {sim }}$ and $\phi_{\mathrm{NiFe}}^{\mathrm{sim}}$ tend to $0^{\circ}$ and $180^{\circ}$, respectively. Because of the toroidal magnetization structure, on the other hand, the effective meron pair's 3D interlayer coupling angle $\delta$ (between $\mathbf{m}^{\mathrm{Co}}$ and $\mathbf{m}^{\mathrm{NiFe}}$ ) may be smaller than the planar coupling angle $\Phi$ (between $\mathbf{m}_{x y}^{\text {Co }}$ and $\mathbf{m}_{x y}^{\mathrm{NiFe}}$ ), as sketched in Fig. 3(b) [49]. 
The 3D magnetization structure of the effective meron pair can be explained in the following way. The dimensions of the ferromagnetic thin-film elements in the trilayer disk would lead to a formation of regular tangential vortices in an isolated case. However, the AFM IEC with significant biquadratic contribution causes a relative canting of the $c$-antisymmetric vortices, which results in the planar convergent or divergent magnetization pattern described. In sample regions at around half the distance between center and edge, the coupling angle matches the theoretical expectations for a continuous film. However, a purely planar convergent or divergent configuration would be associated with extensive magnetic surface and especially volume charges. For this reason, the magnetization tends to align again tangentially when getting close to the edge and the center where the charges would become most relevant. Furthermore, starting from a planar orientation at a certain distance from the center $(>1 / 2 R)$, the magnetization tilts out of plane increasingly towards the central core andwith opposing sign - also towards the edge, which results in the flux-closing magnetization torus described. As this effect is more prominent in regions close to the Rh interface, a vertical $\nabla \mathbf{M}_{z}$ occurs which partially compensates the volume charge generating $\nabla \mathbf{M}_{x y}$. Furthermore, a perpendicular orientation reduces the IEC energy (apart from the core region) as the 3D coupling angle $\delta$ is always smaller than the planar coupling angle $\Phi$. A quantitative view on the 3D toroidal flux closure of magnetic induction can be found in the Supplemental Material [50].

As a consequence of the toroidal magnetization structure, an additional topology is introduced to the vortex pair system. This topology causes the following mutual symmetry constraints with respect to the circulations, polarities, and divergences of the effective meron pair:

$$
p_{1}=p_{2}, \quad c_{1}=-c_{2}, \quad d_{1}=-d_{2} .
$$

While the $c$ state is still independent from $p$ and $d$,

$$
c_{1}=-c_{2}= \pm 1,
$$

there is a topology induced symmetry break enforcing the following relation between $p$ and $d$ :

$$
p_{i}=d_{i} \operatorname{sgn} z_{i}
$$

with the $z$ origin being located at the interlayer position. This means that the core polarities must be aligned along the toroidal flux direction. The latter relation was verified by micromagnetic simulations which show that any $p_{i}=-d_{i} \operatorname{sgn} z_{i}$ configuration relaxes into the stable state proposed. Compared to the $c-p$ correlation which was experimentally observed for single layer vortices [51], the $p-d$ relation for an effective spin meron is strict and universal. The results presented above also imply that spin meron states can only occur in pairs unless a noncollinear exchange, such as the Dzyaloshinskii-Moriya interaction, is present in the ferromagnetic material $[51,52]$.
In summary, this work offers direct proof for the existence of effective spin meron pairs in magnetic trilayers. In the presence of biquadratic IEC, such a pair can even be the magnetic ground state of the system. The 3D structure of an effective meron pair involves significant perpendicular components, which lead to a flux-closing toroidal magnetization distribution. The given topology causes a symmetry break, enforcing the meron cores to be aligned parallel, with the polarity pointing along the torus' orientation. The results of our study provide new insights into the topology and 3D structure of coupled multilayer spin systems. They also might be relevant for possible application concepts involving coupled vortices, such as memory cells or spintorque oscillators. In particular, the substantially enhanced meron core size could be a key factor with respect to spin-torque excitation of vortices.

We would like to thank Aleksandar Puzić for his contribution to this work as well as Riccardo Hertel and Jeffrey McCord for fruitful discussions. We also gratefully acknowledge help from the following people at various stages: Oskar Liedke, Vicki Kühn, Bernd Scheumann, Benjamin Watts, and Julia Dschemuchadse. The STXM experiments were performed using the X07DA (PolLux) beam line at the Swiss Light Source, Paul Scherrer Institut, Villigen, Switzerland. Funding from BMBF (Contract No. 05 KS4WE1/6) is acknowledged.

*s.wintz@hzdr.de

${ }^{\dagger}$ Present address: Fraunhofer-Institut für Photonische Mikrosysteme, 01109 Dresden, Germany.

†Present address: Vacuumschmelze GmbH \& Co. KG, 63450 Hanau, Germany.

${ }^{\S}$ Present address: MAX IV Laboratory, 22363 Lund, Sweden.

[1] F. Bais, Topological Excitations in Gauge Theories; An Introduction From the Physical Point of View, Springer Lecture Notes in Mathematics Vol. 926 (Springer, New York, 1982), p. 107.

[2] L. Fu, C. L. Kane, and E. J. Mele, Phys. Rev. Lett. 98, 106803 (2007).

[3] T. Senthil and M.P. A. Fisher, Phys. Rev. B 63, 134521 (2001).

[4] A. P. Malozemoff and J. C. Slonczewski, Magnetic Domain Walls in Bubble Materials (Academic, New York, 1979).

[5] C. Kittel, Rev. Mod. Phys. 21, 541 (1949).

[6] C. Moutafis, S. Komineas, and J. A. C. Bland, Phys. Rev. B 79, 224429 (2009).

[7] E. Feldtkeller and H. Thomas, Phys. Kondens. Mater. 4, 8 (1965).

[8] T. Shinjo, T. Okuno, R. Hassdorf, K. Shigeto, and T. Ono, Science 289, 930 (2000).

[9] B. E. Argyle, E. Terrenzio, and J. C. Slonczewski, Phys. Rev. Lett. 53, 190 (1984).

[10] S.-B. Choe, Y. Acremann, A. Scholl, A. Bauer, A. Doran, J. Stöhr, and H. A. Padmore, Science 304, 420 (2004).

[11] R.P. Cowburn, D. K. Koltsov, A. O. Adeyeye, M.E. Welland, and D. M. Tricker, Phys. Rev. Lett. 83, 1042 (1999). 
[12] B. Van Waeyenberge, A. Puzic, H. Stoll, K. W. Chou, T. Tyliszczak, R. Hertel, M. Fähnle, H. Brückl, K. Rott, G. Reiss, I. Neudecker, D. Weiss, C. H. Back, and G. Schütz, Nature (London) 444, 461 (2006).

[13] S. Petit-Watelot, J.-V. Kim, A. Ruotolo, R. M. Otxoa, K. Bouzehouane, J. Grollier, A. Vansteenkiste, B. Van de Wiele, V. Cros, and T. Devolder, Nat. Phys. 8, 682 (2012).

[14] S. Bohlens, B. Krüger, A. Drews, M. Bolte, G. Meier, and D. Pfannkuche, Appl. Phys. Lett. 93, 142508 (2008).

[15] V. S. Pribiag, I. N. Krivorotov, G. D. Fuchs, P. M. Braganca, O. Ozatay, J. C. Sankey, D. C. Ralph, and R. A. Buhrman, Nat. Phys. 3, 498 (2007).

[16] A. Ruotolo, V. Cros, B. Georges, A. Dussaux, J. Grollier, C. Deranlot, R. Guillemet, K. Bouzehouane, S. Fusil, and A. Fert, Nat. Nanotechnol. 4, 528 (2009).

[17] V. Sluka, A. Kákay, A. M. Deac, D. E. Bürgler, R. Hertel, and C. M. Schneider, J. Phys. D 44, 384002 (2011).

[18] J. Shibata, K. Shigeto, and Y. Otani, Phys. Rev. B 67, 224404 (2003).

[19] H. Jung, K.-S. Lee, D.-E. Jeong, Y.-S. Choi, Y.-S. Yu, D.-S. Han, A. Vogel, L. Bocklage, G. Meier, M.-Y. Im, P. Fischer, and S.-K. Kim, Sci. Rep. 1, 10 (2011).

[20] K. S. Buchanan, K. Y. Guslienko, A. Doran, A. Scholl, S. D. Bader, and V. Novosad, Phys. Rev. B 72, 134415 (2005).

[21] K. W. Chou, A. Puzic, H. Stoll, G. Schütz, B. Van Waeyenberge, T. Tyliszczak, K. Rott, G. Reiss, H. Brückl, I. Neudecker, D. Weiss, and C. H. Back, J. Appl. Phys. 99, 08F305 (2006).

[22] P. Vavassori, V. Bonanni, A. Busato, D. Bisero, G. Gubbiotti, A. O. Adeyeye, S. Goolaup, N. Singh, C. Spezzani, and M. Sacchi, J. Phys. D 41, 134014 (2008).

[23] J. Wu, D. Carlton, E. Oelker, J.S. Park, E. Jin, E. Arenholz, A. Scholl, C. Hwang, J. Bokor, and Z. Q. Qiu, J. Phys. Condens. Matter 22, 342001 (2010).

[24] J. Kurde, J. Miguel, D. Bayer, J. Sánchez-Barriga, F. Kronast, M. Aeschlimann, H. A. Dürr, and W. Kuch, New J. Phys. 13, 033015 (2011).

[25] S. Wintz, T. Strache, M. Körner, M. Fritzsche, D. Markó, I. Mönch, R. Mattheis, J. Raabe, C. Quitmann, J. McCord, A. Erbe, and J. Fassbender, Appl. Phys. Lett. 98, 232511 (2011).

[26] S. Wintz, T. Strache, M. Körner, C. Bunce, A. Banholzer, I. Mönch, R. Mattheis, J. Raabe, C. Quitmann, J. McCord, A. Erbe, K. Lenz, and J. Fassbender, Phys. Rev. B 85, 134417 (2012).

[27] S. Wintz, C. Bunce, A. Banholzer, M. Körner, T. Strache, R. Mattheis, J. McCord, J. Raabe, C. Quitmann, A. Erbe, and J. Fassbender, Phys. Rev. B 85, 224420 (2012).

[28] O. V. Sukhostavets, G. R. Aranda, and K. Y. Guslienko, J. Appl. Phys. 111, 093901 (2012).

[29] A. A. Awad, A. Lara, V. Metlushko, K. Y. Guslienko, and F. G. Aliev, Appl. Phys. Lett. 100, 262406 (2012).

[30] C. J. Stevenson and J. Kyriakidis, Phys. Rev. B 84, 075303 (2011).

[31] M. Ezawa, Phys. Rev. B 83, 100408(R) (2011).

[32] J. Wu, D. Carlton, J. S. Park, Y. Meng, E. Arenholz, A. Doran, A. T. Young, A. Scholl, C. Hwang, H. W. Zhao, J. Bokor, and Z. Q. Qiu, Nat. Phys. 7, 303 (2011).

[33] M. Yan, H. Wang, and C. Campbell, J. Magn. Magn. Mater. 320, 1937 (2008).
[34] C. Phatak, A. K. Petford-Long, and O. Heinonen, Phys. Rev. Lett. 108, 067205 (2012).

[35] P. Grünberg, R. Schreiber, Y. Pang, M. B. Brodsky, and H. Sowers, Phys. Rev. Lett. 57, 2442 (1986).

[36] J. Unguris, R. J. Celotta, and D. T. Pierce, Phys. Rev. Lett. 67, 140 (1991).

[37] M. Rührig, R. Schäfer, A. Hubert, R. Mosler, J. A. Wolf, S. Demokritov, and P. Grünberg, Phys. Status Solidi A 125, 635 (1991).

[38] J. C. Slonczewski, Phys. Rev. Lett. 67, 3172 (1991).

[39] The disks were patterned along a straight line with structure spacing $>2 R$. X-ray transparent silicon nitride $(t=100 \mathrm{~nm})$ was used as substrate. A thin Cr cap layer preserves the stack from oxidation. Upon the disks, a $\mathrm{Cu}$ microstrip of $t=$ $150 \mathrm{~nm}$ was placed in a second fabrication step for use as an excitation source for future experiments.

[40] S. S. P. Parkin, Phys. Rev. Lett. 67, 3598 (1991).

[41] J. Raabe, G. Tzvetkov, U. Flechsig, M. Böge, A. Jaggi, B. Sarafimov, M. G. C. Vernooij, T. Huthwelker, H. Ade, D. Kilcoyne, T. Tyliszczak, R. H. Fink, and C. Quitmann, Rev. Sci. Instrum. 79, 113704 (2008).

[42] Note that the state of this individual disk is representative for the whole set of virgin structures investigated. However, the relative coupling angle $\Phi$ can slightly vary between different structures.

[43] See Supplemental Material at http://link.aps.org/ supplemental/10.1103/PhysRevLett.110.177201 for details on the measurement of $J_{L}^{f}$ and $J_{Q}^{f}$.

[44] See Supplemental Material at http://link.aps.org/ supplemental/10.1103/PhysRevLett.110.177201 for details on the response of meron pairs to static magnetic fields.

[45] M. R. Scheinfein, http://llgmicro.home.mindspring.com.

[46] Simulation size $\left[\pi \times 1000^{2} \times(50+0.8+50)\right] \mathrm{nm}^{3}$, saturation magnetizations $M_{s}^{\mathrm{NiFe}}=0.74 \times 10^{6} \mathrm{~A} / \mathrm{m}$ and $M_{s}^{\mathrm{Co}}=1.27 \times 10^{6} \mathrm{~A} / \mathrm{m}$, standard exchange constants $A^{\mathrm{NiFe}}=1.05 \times 10^{-11} \mathrm{~J} / \mathrm{m}$ and $A^{\mathrm{Co}}=3.05 \times 10^{-11} \mathrm{~J} / \mathrm{m}$, $J_{Q}=1.05 \mathrm{~mJ} / \mathrm{m}^{2}$, neglected intrinsic anisotropies $K_{u}^{i}=0$. Vacuum permeability was assumed for the interlayer. In order to match the experimental coupling angle of $\Phi \sim 135^{\circ}$, the ratio $J_{L} / J_{Q}$ was set to 1.41 by reducing $J_{L}$ to $-1.51 \mathrm{~mJ} / \mathrm{m}^{2}$ compared to the experimental trilayer value of $-1.95 \mathrm{~mJ} / \mathrm{m}^{2}$. This adaption of $J_{L} / J_{Q}$ reduces the overall saturation field as is expected for roughness induced effects.

[47] T. Gilbert, IEEE Trans. Magn. 40, 3443 (2004).

[48] $\mathrm{NiFe}$ single layer simulation $\left(\pi \times 1000^{2} \times 50\right) \mathrm{nm}^{3}$ on a $256 \times 256 \times 5$ grid. Trilayer simulation of a Co50/ vacuum $0.8 / \mathrm{NiFe} 50(\mathrm{~nm})$ vortex pair with antiferromagnetic circulations and parallel cores $\left[\pi \times 1000^{2} \times(50+0.8+\right.$ $50)] \mathrm{nm}^{3}$ on a $256 \times 256 \times(2+1+2)$ grid. All other parameters were set in accordance with the values given earlier.

[49] See Supplemental Material at http://link.aps.org/ supplemental/10.1103/PhysRevLett.110.177201 for details on the simulated angle distributions.

[50] See Supplemental Material at http://link.aps.org/ supplemental/10.1103/PhysRevLett.110.177201 for quantitative details on the toroidal flux closure.

[51] M.-Y. Im, P. Fischer, K. Yamada, T. Sato, S. Kasai, Y. Nakatani, and T. Ono, Nat. Commun. 3, 983 (2012).

[52] A. N. Bogdanov and U.K. Rössler, Phys. Rev. Lett. 87, 037203 (2001). 